\title{
Electrochemical Preparation and Characterization of a Gold Nanoparticles Graphite Electrode: Application to Myricetin Antioxidant Analysis
}

\author{
Khan Loon Ng, See Mun LeE, Sook Mei KHor, and Guan Huat TaN ${ }^{\dagger}$ \\ Department of Chemistry, Faculty of Science, University of Malaya, 50603 Kuala Lumpur, Malaysia
}

\begin{abstract}
Graphite material is abundantly available from recyclable sources. It possesses a good electrical conductance property, which makes it an attractive material as a working electrode. However, due to a high activation overpotential it has limited applications as compared to other solid metal electrodes. In this present work, we obtained a graphite rod from a used battery, and carried out electrochemical improvements by electro-deposition with gold nanoparticles (AuNPs). The heterogeneous electron transfer rate and electron transfer resistance of the fabricated electrode were improved. The electrode overpotential has shown improvement by $50 \mathrm{mV}$, and the effective surface area has increased by 2 fold. To determine the practicability of the AuNPs/graphite electrode, we used the electrode in the analysis of myricetin. A squarewave voltammetry was used in the analysis, and the detection response increased by 2.5 fold, which suggested an improvement in the electrode sensitivity.
\end{abstract}

Keywords Graphite, gold nanoparticles, electrodeposition, myricetin, anti-oxidant, electrochemical measurement

(Received May 13, 2015; Accepted June 29, 2015; Published October 10, 2015)

\section{Introduction}

Graphite has been known for its electrical conducting properties and chemical inertness. ${ }^{1}$ This has made it an attractive material for working electrodes. It is also abundantly available from recyclable sources, such as used batteries, which can be easily obtained and used as a working electrode. It is estimated that the world market for batteries in the year 2016 will reach USD132 billion, and the production of alkaline batteries in China alone is estimated at $128 \times 10^{8}$ units. $^{2}$ This suggests that used batteries could be potential sources of graphite that can be recycled into working electrodes. In addition, graphite from used batteries possesses ideal dimensions, which fit electrode fabrication with a diameter of 3.0 or $2.0 \mathrm{~mm}$, depending on the battery grade. Unfortunately, a drawback with graphite is often associated with its high activation of overpotential, which impacts on the sensitivity when used in electrochemical analysis. ${ }^{3}$ The chemical structure of graphite comprises $\mathrm{sp}^{2}$ carbon atoms, which are arranged in a honeycomb laminar structure, and positions a free valence electron on its beta carbon, which readily forms the van der Waals interaction. ${ }^{4}$ This unique structure of graphite allows ease of modification on a surface with a metal. ${ }^{1}$ Various deposition procedures of gold nanoparticles (AuNPs) on carbon electrodes have been studied including electro-polymerization, ${ }^{5-8}$ electro-grafting methods ${ }^{9,10}$ and electro-deposition. ${ }^{11-15}$ The latter method provides a much easier, rapid and lower cost procedure ${ }^{13}$ because the preparation is less tedious and involves minimum chemical usage.

$\dagger$ To whom correspondence should be addressed.

E-mail: ghtan@um.edu.my
Several studies on electrochemical applications of used graphite electrodes have been reported. These include the application of pencil graphite in the study of dopamine and uric acid, ${ }^{16}$ a quercetin modified pencil graphite electrode in the study of the electro catalytic oxidation of nicotinamide adenosine dinucleotide, ${ }^{17}$ and a topotecan immobilized pencil graphite electrode for DNA interaction studies. ${ }^{18}$ These studies have successfully demonstrated a low-cost procedure in the fabrication of disposable working electrodes from pencil graphite, which highlight the potential of any surface modification for transforming a graphite material into a sensitive and selective sensor.

Flavonoid compounds extracted from various plants possess anti-oxidant properties, in which it can be studied using an electrochemical method. Myricetin is one of the naturally occurring flavonoids that can be found abundantly in vegetables, such as tomato, cabbage, onion, carrot, spinach and cauliflower. ${ }^{19,20}$ Among the flavonoids, myricetin is one of the few that possess a strong radical scavenging property ${ }^{21}$ with a chemical structure that is similar to that of quercetin. ${ }^{20}$ Studies have shown the significance of myricetin to health, including as an anti-oxidant, therapeutic potential in diabetes mellitus, ${ }^{22}$ and also as an inhibitor in cancer cell proliferation. ${ }^{23}$ The analysis of myricetin can be performed by several analytical techniques, such as liquid chromatography ${ }^{19,24}$ and gas chromatography. ${ }^{25}$ Although these methods are accurate and precise, such analytical procedures often require tedious sample preparation, expensive equipment investment, and are therefore inappropriate for fieldanalysis. In addition, the stability of anti-oxidant compounds is sensitive and can be influenced by various conditions, such as the temperature, environmental oxygen, $\mathrm{pH}$, and light. ${ }^{26,27}$ Therefore, on-field analysis is preferable, which could be 
possible with an electro-chemical method, which is also sensitive, rapid, and lower cost. ${ }^{28}$

In this study, we propose a green, simple and cost-effective procedure for fabricating a graphite working electrode from a used battery. To overcome the high activation overpotential, we performed a surface modification by electrodeposition with AuNPs, which would improve the electrochemical properties of the graphite electrode. The prepared AuNPs graphite electrode was used in the analysis of myricetin.

\section{Experimental}

\section{Chemicals and apparatus}

Britton-Robinson buffer was prepared by mixing an equal molar amount of phosphoric acid, boric acid and acetic acid. All of the chemicals used in the buffer preparation were purchased from Merck (Darmstadt, Germany). A $1000 \mu \mathrm{g} \mathrm{mL}^{-1}$ stock solution of myricetin was prepared from pure powder that was purchased from King Herbs (China) and dissolved in $0.1 \mathrm{~mol} \mathrm{~L}^{-1}$ Britton-Robinson buffer. The prepared myricetin solution was stored in a dark container and kept at $+4^{\circ} \mathrm{C}$. Also, $1.0 \mathrm{mmol} \mathrm{L}{ }^{-1}$ tetrachloroauric acid was prepared from $\mathrm{HAuCl}_{4}$. $\left(\mathrm{H}_{2} \mathrm{O}\right)_{3}$ powder purchased from Sigma Aldrich (Steinheim, Germany), and dissolved in a $0.1 \mathrm{~mol} \mathrm{~L}{ }^{-1} \mathrm{NaNO}_{3}$ solution. A $0.1 \mathrm{~mol} \mathrm{~L}^{-1}$ portion of a potassium ferricyanide $\mathrm{K}_{3} \mathrm{Fe}(\mathrm{CN})_{6}$ solution ( $\mathrm{pH}$ 7) was prepared for electrode characterization. All solutions were prepared using 18.2 $\mathrm{M} \Omega$ deionized water.

All electrochemical measurements were performed using a Metrohm PG Stat 202 (Utrecht, Netherlands). A three-electrode system was employed throughout the experiments, in which a platinum rod was used as a counter electrode, and $\mathrm{Ag} / \mathrm{AgCl}$ ( $3 \mathrm{~mol} \mathrm{~L}^{-1} \mathrm{KCl}$ ) as the reference electrode; both electrodes were purchased from BASi (USA). Morphology analysis on the electrode surface was performed using a Hitachi field emission scanning electron microscope (Model SU8220, Japan).

\section{Fabrication of graphite electrode}

A graphite rod was carefully removed from a used AA grade battery. The rod was cleaned with ultrapure water (submerged), followed by 20-min sonication. The cleaned graphite rod was dried in an oven for 2 days at $130^{\circ} \mathrm{C}$, and the tip was polished with emery paper, grade P130, then followed by grades P1000 and P2000. A dielectric PTFE tape was used to insulate the lateral exposure of graphite rod, and finally the rod was inserted into a $3 \mathrm{~mm}$ diameter PVC tube. The fabricated graphite electrode was polished with alumina slurry consisting of $1.0 \mu \mathrm{m}$ particles, and then followed by particle sizes of 0.30 and $0.05 \mu \mathrm{m}$ on a Texmet pad. After polishing, the electrode was cleaned by sonicating in ethanol for $5 \mathrm{~min}$, followed by ultrapure water. The fabricated graphite electrode was kept at room temperature until further use.

Electro-deposition and activation of gold nanoparticles on a graphite electrode

The electrodeposition procedure used was based on a proposed method by Hezard et al., ${ }^{13}$ but with some modification. AuNPs deposition on the graphite electrode was obtained by cyclic voltammetry $(\mathrm{CV})$ scanning of the graphite electrode in a solution of $0.1 \mathrm{mmol} \mathrm{L}^{-1}$ of tetrachloroauric acid at $\mathrm{pH}$ 3. The $\mathrm{CV}$ was scanned in the potential range of 0.90 to $-0.30 \mathrm{~V}$ with scan rates and potential steps of $0.05 \mathrm{~V} \mathrm{~s}^{-1}$ and $0.0025 \mathrm{~V}$, respectively. A total of 6 preparations at 4, 8, 12, 16, 20 and 24 deposition cycles numbers were performed.

The electrodes were electrochemically activated by performing a CV scan in a solution of $0.5 \mathrm{~mol} \mathrm{~L}^{-1}$ sulfuric acid. The $\mathrm{CV}$ was scanned from 0.20 to $1.60 \mathrm{~V}$ with a scan rate of $0.10 \mathrm{~V} \mathrm{~s}^{-1}$ and potential steps of $0.0025 \mathrm{~V}$. A total of 20 repetition cycles were performed for each activation. The surface-modified electrode was designated to be an AuNPs/graphite electrode.

\section{Electrochemical characterization and morphology analysis of AuNPs/graphite}

Activated AuNPs/graphite was characterized with $\mathrm{CV}$ and electro-impedance spectroscopy (EIS). Both experiments were performed in a $1.0 \mathrm{mmol} \mathrm{L}^{-1}$ ferricyanide solution, with the $\mathrm{CV}$ scanned from -0.15 to $0.65 \mathrm{~V}$ across a range of scan rates from 0.02 to $0.80 \mathrm{~V} \mathrm{~s}^{-1}$. An EIS experiment was performed at $0.20 \mathrm{~V}$ with a frequency range from $1.00 \mathrm{KHz}$ to $0.10 \mathrm{~Hz}$.

The tip of the surface modified graphite electrode was disengaged with a pair of cutters. The surface of the tip was positioned up-ward, and the bottom was adhered onto an FESEM sample platform; a low-vacuum mode was used, and the electron power was set at $30 \mathrm{KeV}$.

\section{Electrochemical analysis of myricetin using a AuNPs/graphite electrode}

The myricetin assay was performed using a square-wave voltammetry (SWV) technique in a deaerated Britton-Robinson buffer; with the applied potential ranging from -0.20 to $0.80 \mathrm{~V}$, frequency and the amplitude were $50 \mathrm{~Hz}$ and $25 \mathrm{mV}$, respectively. A standard calibration curve of myricetin was constructed using the standard addition method with a $2.0 \mu \mathrm{g} \mathrm{mL}^{-1}$ increment in each addition. The limit of detection (LOD) and optimum $\mathrm{pH}$ condition of myricetin was determined.

\section{Results and Discussion}

Electrodeposition of gold nanoparticles on a graphite electrode

The CV of the gold nanoparticles deposition on a graphite electrode is illustrated in Fig. 1(a), with only the first, third and last CV scans being shown in Fig. 1(a). In a reverse scan from 0.90 to $-0.30 \mathrm{~V}$, a cathodic peak was observed. This peak was associated with the reduction of gold(III) to elemental gold. ${ }^{13,29}$ When the $\mathrm{CV}$ results were analyzed from the 1 st to the subsequent 3rd and 10th $\mathrm{CV}$, the peak shifted to a more positive potential, with the first scan and the 10th scan being recorded at 0.55 and $0.71 \mathrm{~V}$, respectively. No significant peak potential shift was observed after the 10th scan. The positive potential shift could be attributed to a favorable thermodynamic deposition of gold on the metal substrate (nucleation process) instead of on the carbon substrate, since it required more adsorption energy. ${ }^{4}$ Based on this evaluation, the electrodeposition of gold at the 4th and 8th deposition cycles could result in a lesser nucleation process with smaller and more consistent particle sizes, as compared to the higher deposition cycles. This was because, at the 10th deposition cycle and above, it would only favor the nucleation process with continued growth in the particle size, and thus leading to a wider size distribution. This observation was confirmed by a morphology analysis with a field-emission scanning electron microscope. Figure 1(b) images B, C and D illustrate the SEM results of the AuNPs graphite surface prepared at the 8th, 16th and 24th deposition cycle. The size of the AuNPs was measured, which corresponded to diameter of 75,110 , and 180 , respectively. A substantial increment of the AuNPs size from the 8 to 24 deposition cycle suggested that the nucleation process is favorable at higher deposition cycles. 
(a)



(b)
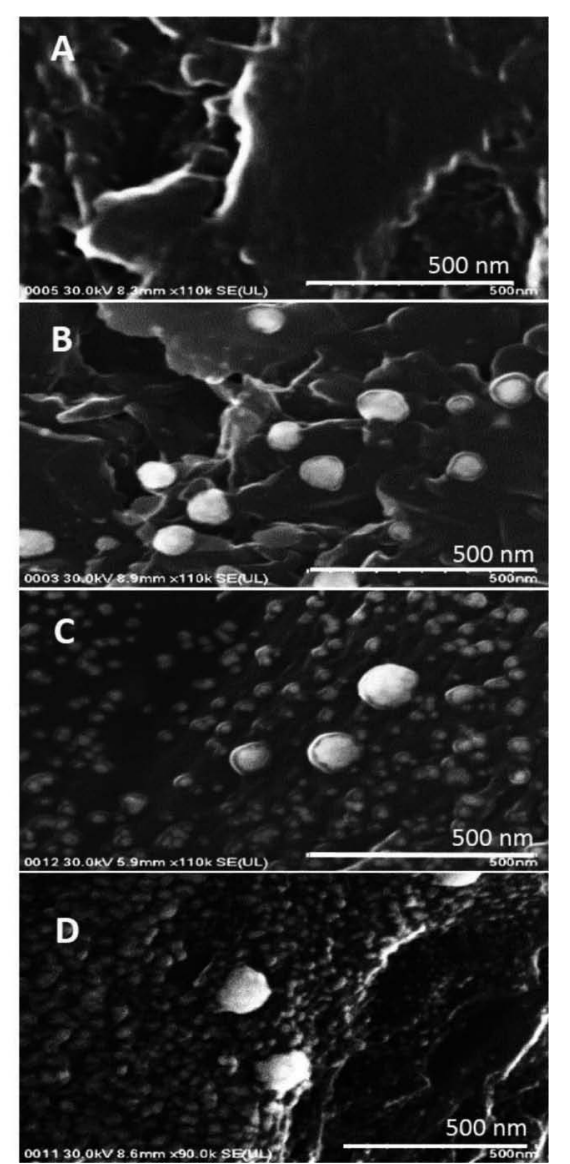

Fig. 1 (a) Cyclic voltammetry of gold nanoparticles deposition on a graphite electrode. The dotted, thick and thin solid lines correspond to the first, third and tenth deposition cycles (b) Scanning electron microscope of bare graphite (images A) and AuNPs graphite electrode prepared at the 8th (images B), 16th (images C) and 24th (images D) deposition cycles.

Activation of an AuNPs/graphite electrode

An AuNPs/graphite electrode was activated using $20 \mathrm{CV}$ scans in $0.5 \mathrm{~mol} \mathrm{~L}^{-1}$ sulfuric acid. In the forward $\mathrm{CV}$ scan from 0.20 to $1.60 \mathrm{~V}$, a large peak was observed in the region of 1.30 to $1.60 \mathrm{~V}$. According to Yamamoto et al., this peak could likely be due to the formation of $\mathrm{Au}(\mathrm{III})$ hydroxide on the graphite surface, $1^{1,13,29,30}$ in the reverse CV scan from 1.60 to $0.20 \mathrm{~V}$, a peak was observed at a potential of $0.87 \mathrm{~V}$, which corresponded to stripping of the $\mathrm{Au}$ oxide..$^{29,30}$ By comparing the 1st with the 20th CV scans, a decrease in the peak current at the oxidation region was observed, which suggested a suppression of $\mathrm{Au}$ oxide formation on the graphite surface.

Morphology characterization by a field-emission scanning electron microscope (FESEM)

The morphology of the bare and gold nanoparticles graphite electrode was characterized using FESEM. Figure S1 (Supporting Information) illustrates the SEM images of the bare and gold nanoparticles graphite electrode, which were prepared at the 8th, 16th, and 24th deposition cycles. The AuNPs/ graphite electrodes prepared at the 16th (image C) and 24th (image D) deposition cycle showed a more homogenous distribution of the nanoparticles at $30 \mathrm{~K}$ magnification. In contrast, to the 8th (image B) deposition cycle, the gold nanoparticles distribution was less consistent with a much narrower size distribution. With greater SEM magnification at 110K (Images C and D), as shown in Fig. 1(b), a mixture of small and large nanoparticles could be observed with the smaller nanoparticles on the electrode prepared at the 16th and 24th deposition cycles measuring between $35-65 \mathrm{~nm}$ and $10-$ $100 \mathrm{~nm}$, respectively. The shapes of the smaller particles are irregular, while the larger particles are spherical.

Electrochemical characterization of an AuNPs/graphite electrode by cyclic voltammetry

$$
I_{\mathrm{p}}=2.69 \times 10^{5} \times n^{\frac{3}{2}} \times A C D^{\frac{1}{2}} v^{\frac{1}{2}}
$$

The effective surface area $\left(A_{\text {eff }}\right)$ is calculated using the Randless-Sevcik equation (Eq. (1)), where $I_{\mathrm{p}}, n, A, C, D$ and $v$ correspond to the peak current (A), number of electron exchanges, effective surface area $\left(\mathrm{cm}^{2}\right)$, concentration $(\mathrm{mol} \mathrm{cm}$ $3)$, diffusion coefficient $\left(\mathrm{cm} \mathrm{s}^{-1}\right)$ and scan rate $\left(\mathrm{V} \mathrm{s}^{-1}\right)$. Tables S1 - S3 (Supporting Information) summarizes the effective surface area of bare graphite, inactivated AuNPs/graphite and activated AuNPs/graphite electrode, respectively. The effective surface area of activated AuNPs/graphite showed a much larger surface area when compared to their bare graphite counterpart, with the activated AuNPs/graphite electrode prepared at 16th deposition cycles having the largest effective surface area of $0.165 \mathrm{~cm}^{2}$, in comparison to its bare graphite at $0.048 \mathrm{~cm}^{2}$. Figure S2(a) (Supporting Information) illustrates the surfacearea trend across different deposition cycles of the graphite 

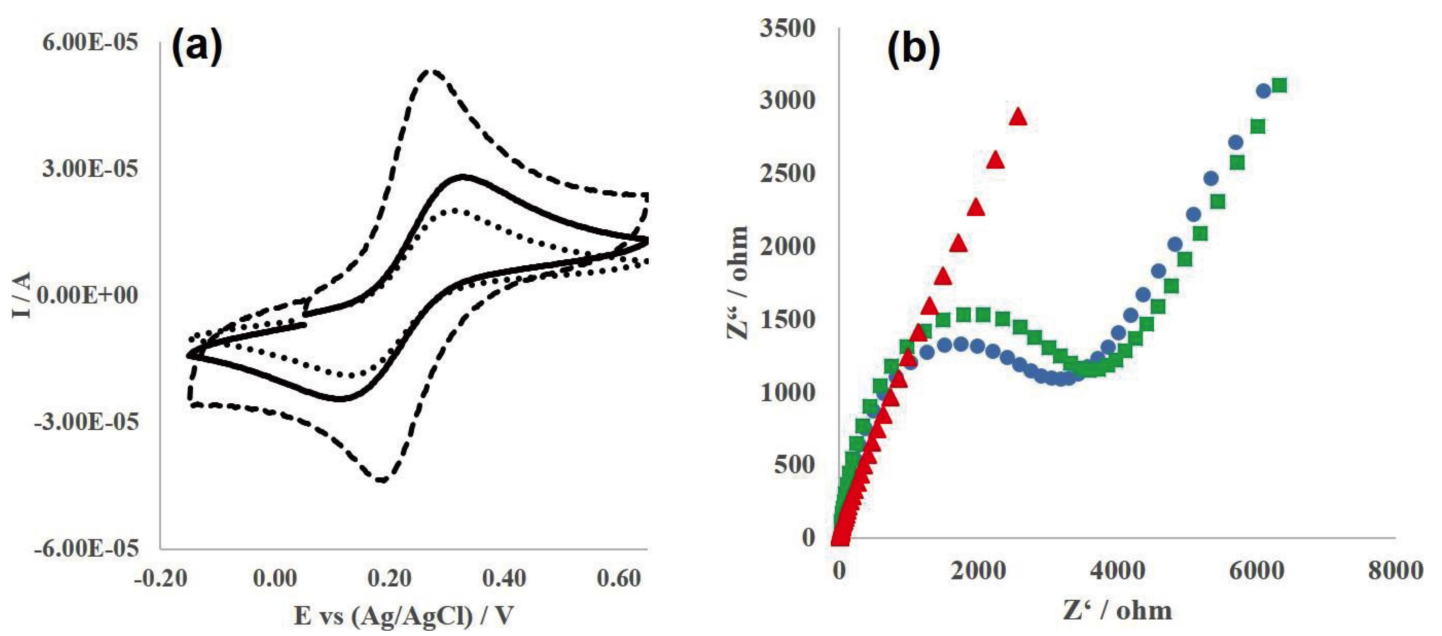

Fig. 2 (a) Cyclic voltammetry of activated (dash line) and an inactivated (dot line) AuNPs/graphite electrode prepared at the 16th deposition cycles. The solid line represent the bare graphite (b) Nyquist plot of bare graphite (sphere marker), inactivated AuNPs/graphite (square marker) and activated AuNPs/graphite (triangle marker) that prepared by the 16th deposition cycles. The experiment was performed in a $0.1 \mathrm{~mol} \mathrm{~L}^{-1}$ ferricyanide solution.

electrodes. The effective surface area of the inactivated AuNPs/ graphite electrodes is the same as that of the bare graphite electrodes. This suggests that the inactivated AuNPs/graphite could be impeded by the Au oxide nanoparticles. Upon activation in an acidic solution by multiple CV scanning, the effective surface area of the activated AuNPs/graphite electrodes was expanded, due to a reduction of the gold oxide to gold.

$$
I_{\mathrm{p}}=n^{2} f^{2} A \Gamma \frac{v}{4 R T}
$$

The surface coverage of the AuNPs on graphite can be calculated using Eq. (2), where, $I_{\mathrm{p}}, n, F, A, \Gamma, R, V$, and $T$ correspond to the peak current (A), number of electrons, Faraday constant $\left(\mathrm{C} \mathrm{mol}^{-1}\right)$, effective surface area $\left(\mathrm{cm}^{2}\right)$, surface coverage $\left(\mathrm{cm}^{-2}\right)$, gas constant $\left(\mathrm{J} \mathrm{mol}^{-1} \mathrm{~K}^{-1}\right)$, scan rate $\left(\mathrm{V} \mathrm{s}^{-1}\right)$ and temperature (K). In this study, the surface coverage was used as a method to evaluate gold nanoparticles deposition. Based on the finding at the 16th deposition cycle, the surface coverage was the highest at $6.01 \times 10^{-10} \mathrm{~cm}^{-2}$. At subsequent depositions above 16 cycles, the surface coverage of the gold started to decrease. This observation could be a result of AuNPs nucleation process instead of deposition on the bare graphite surface. The results of the gold surface coverage in the various AuNPs/graphite deposition cycles are summarized in Table S3 (Supporting Information).

$$
E_{\mathrm{p}}=E_{0}-\frac{2.303 R T}{\alpha n F} \log \frac{R T K_{\mathrm{et}}}{\alpha n f}+\frac{2.303 R T}{\alpha n f} \log v
$$

The heterogeneous electron transfer rate $\left(k_{\mathrm{s}}\right)$ of an AuNPs/ graphite electrode was calculated using the Laviron equation (Eq. (3)), where, $E_{\mathrm{p}}, E_{0}, R, T, n, F$ and $v$ correspond to the peak potential $(\mathrm{V})$, formal potential $(\mathrm{V})$, gas constant $\left(\mathrm{J} \mathrm{mol}^{-1} \mathrm{~K}^{-1}\right)$, temperature $(\mathrm{K})$, number of electrons, Faraday constant $\left(\mathrm{C} \mathrm{mol}^{-1}\right)$ and scan rate $\left(\mathrm{V} \mathrm{s}^{-1}\right)$. The term $\alpha$ is a dimensionless constant that measures the symmetry of the energy barrier of the redox reaction. ${ }^{12}$ The ks value of a bare graphite, inactivated AuNPs/graphite and activated AuNPs/graphite are summarized in Tables S1-S3 (Supporting Information). Overall, the heterogeneous electron-transfer rate of the activated AuNPs/ graphite is the highest as compared to its bare graphite electrode counterpart. The electrode prepared at the 24th deposition cycles showed the highest ks value with a rate constant of $12.25 \mathrm{~s}^{-1}$; this was followed by an electrode prepared at the 16th deposition cycles with $k_{\mathrm{s}}$ of $9.89 \mathrm{~s}^{-1}$. From Fig. S2(b) (Supporting Information), the inactivated AuNPs/graphite has the lowest $k_{\mathrm{s}}$ value as compared to that of bare graphite. This can be explained by the Au oxide surface, which impedes the electron-transfer process.

Overall, after activation, the electrode prepared at the 16th deposition cycles is found to have the optimum electrochemical properties. The electron-transfer efficiency of this electrode was further evaluated with $\mathrm{CV}$; the result is illustrated in Fig. 2(a). The potential difference $(\Delta P)$ between the anodic and cathodic peak of the activated electrode (16th deposition cycles) is separated by $78 \mathrm{mV}$. This value is close to the theoretical value of $59 \mathrm{mV}$ in a Nernstian reversible system, and hence, suggesting a more efficient electron-transfer process as compared to the values of 161 and $183 \mathrm{mV}$ obtained by using the inactivated AuNPs graphite and bare graphite, respectively. The peak separation of the electrode (activated 16th deposition cycles) was also monitored throughout a range of scan rates from 0.02 to $1.25 \mathrm{~V} \mathrm{~s}^{-1}$. The results are summarized in Table S4 (Supporting Information), which suggest that the electron transfer efficiency of the activated AuNPs/graphite (16th deposition cycles) could be attained up to a scan rate of $1.00 \mathrm{~V} \mathrm{~s}^{-1}$.

The electro-deposition of AuNPs on the graphite electrode also exhibited an improvement in the electrode overpotential. Figure 2(a) illustrates the $\mathrm{CV}$ of the ferri/ferro cyanide redox process that was performed using the activated AuNPs graphite (16th deposition cycles) and bare graphite electrode. The results showed a decrease in the anodic peak potential from $0.32 \mathrm{~V}$ (solid line) to $0.27 \mathrm{~V}$ (dotted line). The reduction in the anodic peaks potential by $0.05 \mathrm{~V}$ suggests an improvement in the overpotential after 16 cycles of AuNPs deposition. 

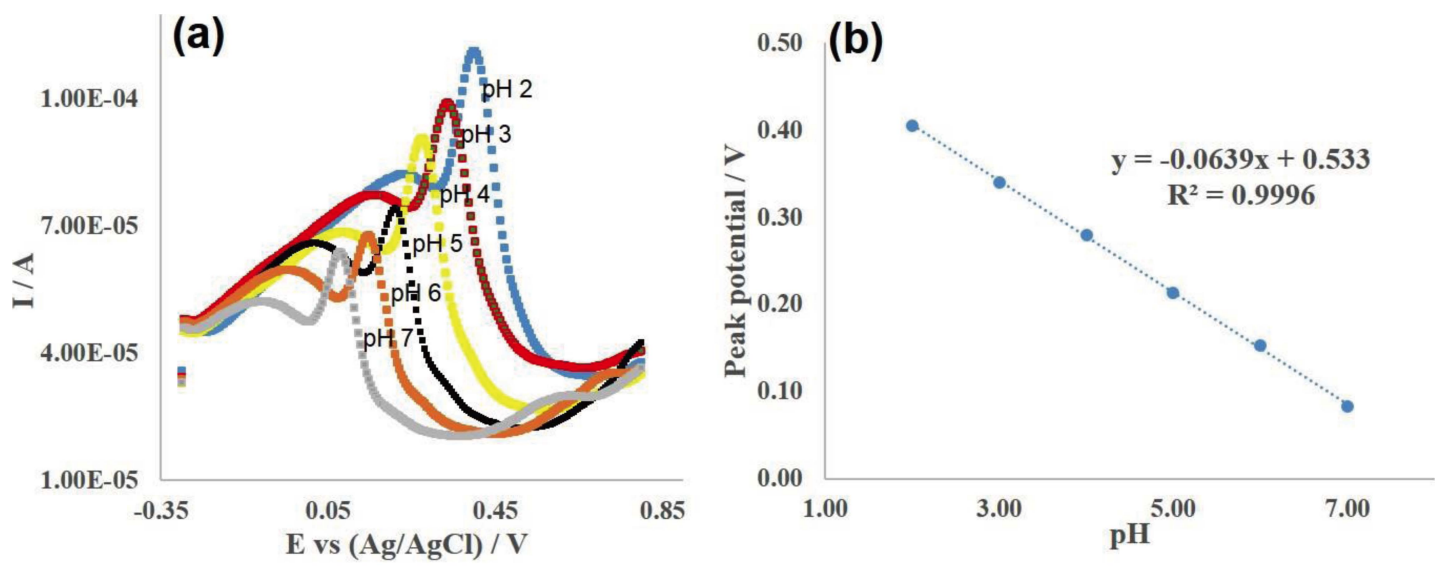

(c)<smiles>O=c1c(O)c(-c2cc(O)c(O)c(O)c2)oc2cc(O)cc(O)c12</smiles><smiles></smiles><smiles></smiles>

Fig. 3 (a) Square-wave voltammetry of $6 \mu \mathrm{g} \mathrm{mL}^{-1}$ myricetin measured at $\mathrm{pH} 2, \mathrm{pH} 3, \mathrm{pH} 4, \mathrm{pH} 5, \mathrm{pH}$ 6 and $\mathrm{pH} 7$ in a $0.1 \mathrm{~mol} \mathrm{~L}^{-1}$ Britton-Robinson buffer. (b) Graph of the myricetin peak potential versus the $\mathrm{pH}$. (c) Schematic illustration of myricetin oxidation and reduction (protonation).

Electrochemical characterization of an AuNPs/graphite electrode by electro impedance spectroscopy (EIS)

The Randless-circuit model is used in the EIS study of a fabricated AuNPs/graphite electrode. Figure S3(b) (Supporting Information) illustrates the Randless-circuit model, in which 4 components of $R_{\text {sol }}$ (Resistance of electrolyte), $R_{\mathrm{ct}}$ (charge transfer resistance), $C_{\mathrm{dl}}$ (double layer capacitance) and $W$ (Warburg's element) are connected in series or parallel manner. The results of the Randless-circuit fitting are summarized in Tables S1 - S3 (Supporting Information). Based on the results at the 16th deposition cycles, the $R_{\mathrm{ct}}$ values for the bare graphite, inactivated AuNPs/graphite and activated AuNPs/graphite, are fitted at 2450, 2840, and $156 \Omega$, respectively. This suggested that, after activation, the AuNPs/graphite electrode showed the lowest electron transfer resistance.

This result is supported by a Nyquist plot, in which the diameter of the semi-circle portion corresponds to the electrontransfer resistance. From Fig. 2(b), the inactivated AuNPs/ graphite has a semi-circle diameter that is comparable to that of bare graphite electrodes. In contrast to the activated AuNPs/ graphite, a linear line is observed instead of a semi-circle, which suggests that after activation, the electrode exhibited a lower electron-transfer resistance.

Figure S3(a) (Supporting Information) illustrates the $C_{\mathrm{dl}}$ properties of activated AuNPs/graphite against the deposition cycle number. The $C_{\mathrm{dl}}$ values of the AuNPs/graphite increase from 4 to 16 cycles, after which the values start to decrease. The $C_{\mathrm{dl}}$ of the AuNPs/graphite electrode that was prepared at 16th deposition cycles has the highest values after activation. This could be attributed to the higher effective surface area and roughness, in contrast to the other electrode prepared at different deposition cycles. The surface roughness factor of the activated AuNPs/graphite electrode is summarized in Table S5 (Supporting Information). This observation was further confirmed with SEM images C and D of Fig. 1(b), whereby the surface at 16th deposition cycle is rougher in comparison to the 24th deposition cycle. The impact of the double layer capacitance on the electrode application can be observed in Fig. 4(c), in which the background current of the myricetin analysis was much higher than that of the bare graphite. In order to determine the impact of the double-layer capacitance to the electrode performance, the capacitive impedance of the electrode was studied as postulated by Pajkossy et al. ${ }^{31}$

Generally, an ideal polarizable electrode should exhibit an ideal capacitive impedance, ${ }^{32}$ which can be determined using a constant phase element (CPE), i.e. the power-law of frequency of capacitance $\left[\mathrm{C}\left(\mathrm{w}^{\mathrm{N}}\right)\right]$. An $\mathrm{N}$ value that is close to 1 denoted that the electrode exhibits an ideal capacitive impedance. ${ }^{31,32}$ The CPE of the AuNPs/graphite electrode prepared at different deposition cycles were determined with the Randless-CPE circuit model; the results are summarized in Table S5 (Supporting Information). From the results, the $\mathrm{N}$-value of the electrode prepared at the 16th deposition cycles is 0.88 . Generally, the CPE values of most solid electrodes are in the range of 0.70 to $0.90 .^{32}$ Although, the high surface area and roughness of the AuNPs/graphite could impact on the capacitive impedance, the CPE fitting suggests that AuNPs/graphite prepared at the 16th deposition cycles was closer to the ideal condition.

From the EIS characterization, an AuNPs graphite electrode prepared at the 16th deposition cycles is found to be the optimum, with a lower electron-transfer resistance after activation. Therefore, based on the EIS and CV data, the activated AuNPs/graphite electrode prepared at the 16th deposition cycles was selected for further studies.

Electrochemical analysis of myricetin using an AuNPs/graphite electrode

A square-wave voltammetry (SWV) method was used in myricetin analysis, and the AuNPs/graphite prepared at the 16th deposition cycle was used as the working electrode. Figure 3(a) illustrates the SWV of myricetin at various $\mathrm{pH}$ values using the 


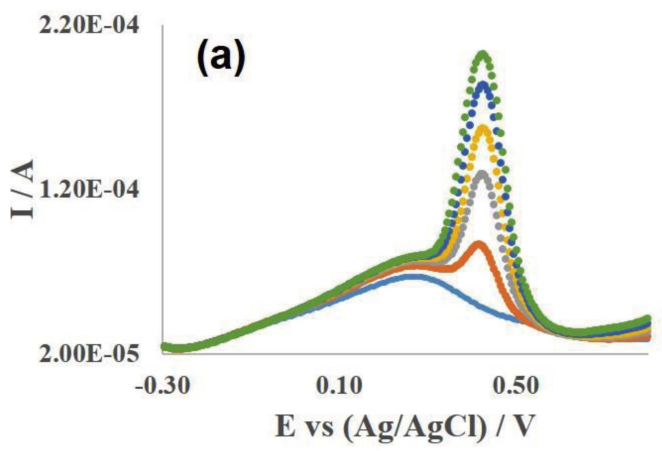

(c)

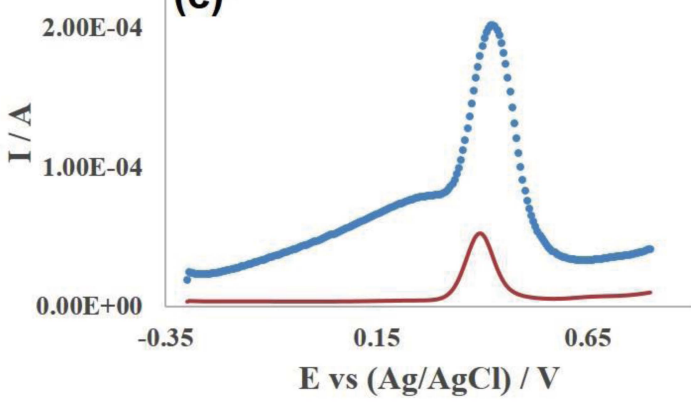

$2.0 \mathrm{E}-05$ (b)

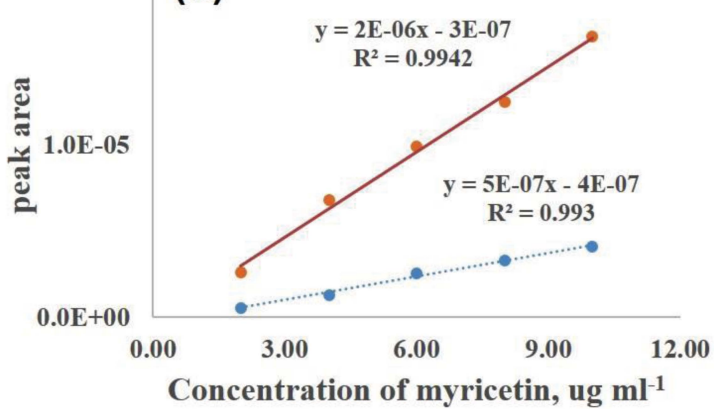

(d)

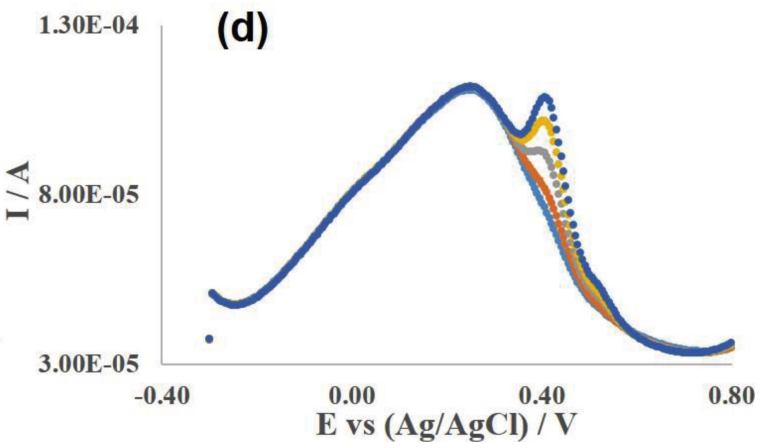

Fig. 4 (a) Square-wave voltammogram (SWV) of myricetin at blank, 2.0, 4.0, 6.0, 8.0 and $10.0 \mu \mathrm{g} \mathrm{mL}^{-1}$ of myricetin standard. (b) Calibration curve of the peak area versus concentration of myricetin. The solid and dotted lines correspond to the AuNPs graphite (16th deposition cycles) and bare graphite working electrodes. (c) SWV of $10.0 \mu \mathrm{g} \mathrm{mL}-1$ myricetin using AuNPs graphite prepared at 16th deposition cycles (dotted line) and bare graphite (solid line). (d) SWV of myricetin with the concentration increment order corresponding to $0.2,0.4,0.6,0.8$ and $1.0 \mu \mathrm{g} \mathrm{mL}^{-1}$.

AuNPs/graphite prepared at the 16th deposition cycles. The peak potential of myricetin shifted to a more negative potential with increasing $\mathrm{pH}$ values. The shift in the peak potential with the $\mathrm{pH}$ could be attributed to the difference in the ratio between the protonated myricetin in the bulk solution and un-protonated species on the electrode surface. Therefore, with a lower $\mathrm{pH}$, the concentration of protonated myricetin in the solution will be much greater, and hence increases the tendency of the protonated myricetin to diffuse, resulting in the peak potential shifting to a more positive potential (i.e. easier for oxidation). ${ }^{33}$ A graph of the peak potential against the $\mathrm{pH}$ was plotted as shown in Fig. 3(b), resulting in a linear correlation between the potential and the $\mathrm{pH}$ with a linear regression and slope value of 0.9996 and $0.0639 \mathrm{~V}$, respectively. From the Nernst equation, the slope of the potential $(\mathrm{V})$ versus $\mathrm{pH}$ is equivalent to $0.0591 \mathrm{~V} / n$, where $n$ is equal to the number of electrons transferred. Therefore, this showed that a single electron and proton were transferred in the myricetin oxidation process, which could be ascribed to the pyrogallol group. ${ }^{33}$ Figure 3(c) illustrates the possible schematic reaction of the myricetin oxidation. The slope value is also in agreement with the result reported by Kormorsky-Lovric et al., in which the slope value obtained in their work was $0.06 \mathrm{~V}$. From Fig. 3(a), the myricetin peak current is highest at $\mathrm{pH} 2$, suggesting an optimum $\mathrm{pH}$ condition for the quantitative analysis of myricetin. This $\mathrm{pH}$ condition was selected for further studies of myricetin.

Figure 4(a) illustrates the SWV of myricetin at various concentrations with the myricetin peak potential at $0.43 \mathrm{~V}$ (versus $\mathrm{Ag} / \mathrm{AgCl}, 3 \mathrm{M} \mathrm{KCl}$ ). A calibration curve of the peak area against the myricetin concentration is shown in Fig. 4(b). The linear regression of the calibration curve was greater than
0.99 , with the linearity range being from 2.0 to $10.0 \mu \mathrm{g} \mathrm{mL}^{-1}$. The experiment was repeated using bare graphite as the working electrode. The calibration curves of the bare graphite and AuNPs/graphite electrodes are compared in Fig. 4(b). The results suggest that the AuNPs/graphite (16 deposition cycles) electrode was more sensitive toward myricetin with a higher response factor (slope), as compared to the bare graphite electrode. By comparing the results from 4(a), 4(b) and 4(c), the peak current of myricetin measured by the AuNPs/graphite electrode showed a 2.5-fold increase in the current, as compared to values obtained from the bare graphite electrode. This could be due to the large surface area, and also the fast electrontransfer rate of the AuNPs/graphite electrode, thus suggesting an improvement in the electrode sensitivity.

The LOD of myricetin is determined by the serial dilution of a $1.0 \mathrm{ppm}$ standard. Figure 4(d) illustrates the SWV of the myricetin peak at concentrations of 0.2 to $1.0 \mu \mathrm{g} \mathrm{mL}^{-1}$. The LOD of myricetin was found at $0.4 \mu \mathrm{g} \mathrm{mL}^{-1}$, and the recovery study is summarized in Table S6 (Supporting Information). At a concentration of $0.4 \mu \mathrm{g} \mathrm{mL}^{-1}$, the percentage of myricetin recovered was less than $90.0 \%$; however, at a concentration of $0.6 \mu \mathrm{g} \mathrm{mL}^{-1}$ and above the recovery of myricetin was greater than $90.0 \%$.

\section{Conclusions}

In this study, we successfully devised a simple, green and costeffective procedure in fabricating a gold nanoparticles graphite electrode from a used battery. The effective surface area, heterogeneous electron transfer rate, electrode overpotential and 
electron-transfer resistance of the AuNPs/graphite electrode were improved when compared to its counterpart bare graphite electrode. It can be inferred that at the 16th deposition cycle of $0.1 \mathrm{mM}$ tetrachloroauric acid, the performance of the AuNPs/ graphite electrode reached an optimum. At this preparation condition, the gold nanoparticles size was measured to be in the range of $35-65 \mathrm{~nm}$, and was homogenously distributed on the graphite surface.

The fabricated AuNPs/graphite electrode was used in the determination of myricetin. The data showed an improvement in the myricetin detection, which suggested an enhancement in the electrode sensitivity by 2.5 fold due with gold nanoparticles surface modification. A square-wave voltammetry method was successfully optimized for the determination of myricetin in Britton-Robinson buffer at $\mathrm{pH}$ 2. The anodic peak current was proportional to the concentration of myricetin in the range from 0.6 to $10.0 \mu \mathrm{g} \mathrm{mL}^{-1}$, with an LOD and limit of quantification (LOQ) of 0.4 and $0.6 \mu \mathrm{g} \mathrm{mL} \mathrm{m}^{-1}$, respectively.

\section{Acknowledgements}

This work was financially supported by the University of Malaya Research Grant (UMRG-Programme RP012C-14SUS), and the Fundamental Research Grant Scheme (FRGS) from the Ministry of Higher Education of Malaysia (MOHE) FP0142013A and FP058-2014A.

\section{Supporting Information}

Scanning electron microscope image at $30 \mathrm{~K}$ magnifiction (Fig. S1), Effective surface area and heterogeneous electron transfer rate trend accross deposition cycle (Fig. S2), Randless circuit (Fig. S3), electrochemical characterization of bare and AuNPs/graphite electrode (Tables S1, S2, and S3), peak potential of AuNPs/graphite versus scan rates (Table S4), surface roughness factor(R) and CPE fitting (Table S5), percentage recoveries of myricetin (Table S6). This material is available free of charge on the Web at http://www.jsac.or.jp/ analsci/.

\section{References}

1. L. Moreno-Baron, A. Merkoçi, and S. Alegret, Electrochim. Acta, 2003, 48, 2599.

2. M. Sun, X. Yang, D. Huisingh, R. Wang, and Y. Wang, J. Cleaner Prod., 2015, 1.

3. S. Wring and J. P. Hart, Analyst, 1992, 117, 1215.

4. D. Appy, H. Lei, C. Wang, M. C. Tringides, D. Liu, J. W. Evans, and P. A. Thiel, Prog. Surf. Sci., 2014, 89, 219.

5. X. Huang, Y. Li, Y. Chen, and L. Wang, Sens. Actuators, B, 2008, 134, 780.

6. N. Li, T. Lei, Y. Liu, Y. He, and Y. Zhang, Trans. Nonferrous Met. Soc. China, 2010, 20, 2314.
7. J. Li and X. Lin, Sens. Actuators, B, 2007, 126, 527.

8. N. German, A. Ramanavicius, J. Voronovic, and A. Ramanaviciene, Colloids Surf., A, 2012, 413, 224.

9. K. Jayakumar, R. Rajesh, V. Dharuman, R. Venkatasan, J. H. Hahn, and S. K. Pandian, Biosens. Bioelectron., 2012 31, 406.

10. D. Bélanger and J. Pinson, Chem. Soc. Rev., 2011, 40, 3995.

11. A. Maringa, E. Antunes, and T. Nyokong, Electrochim. Acta, 2014, 121, 93.

12. N. German, A. Ramanavicius, and A. Ramanaviciene, Sens. Actuators, B, 2014, 203, 25.

13. T. Hezard, K. Fajerwerg, D. Evrard, V. Collière, P. Behra, and P. Gros, J. Electroanal. Chem., 2012, 664, 46.

14. Y. Hu, Y. Song, Y. Wang, and J. Di, Thin Solid Films, 2011, $519,6605$.

15. L. Wang, W. Mao, D. Ni, J. Di, Y. Wu, and Y. Tu, Electrochem. Commun., 2008, 10, 673.

16. E. Alipour, M. Reza, and A. Saadatirad, Electrochim. Acta, 2013, 91, 36.

17. Y. Dilgin, B. Kızılkaya, D. G. Dilgin, H. I. Gökçel, and L. Gorton, Colloids Surf., B, 2013, 102, 816.

18. G. Congur, A. Erdem, and F. Mese, Bioelectrochemistry, 2015, 102, 21.

19. B. Sultana and F. Anwar, Food Chem., 2008, 108, 879.

20. J. H. Huang, C. C. Huang, J. Y. Fang, C. Yang, C. M. Chan, N. L. Wu, S. W. Kang, and C. F. Hung, Toxicol. InVitro, 2010, 24, 21

21. M. Abou Samra, V. S. Chedea, A. Economou, A. Calokerinos, and P. Kefalas, Food Chem., 2011, 125, 622.

22. Y. Li and Y. Ding, Food Sci. Hum. Wellness, 2012, 1, 19.

23. K. Shiomi, I. Kuriyama, H. Yoshida, and Y. Mizushina, Food Chem., 2013, 139, 910.

24. G. Flores and M. Luisa, J. Food Compos. Anal., 2015, 39, 55.

25. A. Kumar, A. K. Malik, and D. K. Tewary, Anal. Chim. Acta, 2009, 631, 177.

26. I. Ioannou, I. Hafsa, S. Hamdi, C. Charbonnel, and M. Ghoul, J. Food Eng., 2012, 111, 208.

27. V. K. Ananingsih, A. Sharma, and W. Zhou, Food Res. Int., 2013, 50, 469.

28. I. Gabriela, A. C. Bizgan, D. Elena, M. Buleandra, H. Basaga, A. A. Ciucu, Z. Moldovan, and I. Adriana, Food Chem., 2015, 173, 1059.

29. Y. Yamamoto, H. Shiigi, and T. Nagaoka, Electroanalysis, 2005, 17, 2224.

30. G. Gotti, K. Fajerwerg, D. Evrard, and P. Gros, Electrochim. Acta, 2014, 128, 412.

31. T. Pajkossy, Solid State Ionics, 2005, 176, 1997.

32. Z. Kerner and T. Pajkossy, Electrochim. Acta, 2000, 46, 207.

33. S. Martinez, L. Valek, Z. Petrovic, M. Metikos-Hukovic, and J. Piljac, J. Electroanal. Chem., 2005, 584, 92.

34. S. Komorsky-Lovric and I. Novak, Electochim, Acta, 2013, 98, 153. 